\title{
Comparison of In Vitro Killing Activity of Rezafungin, Anidulafungin, Caspofungin, and Micafungin against Four Candida auris Clades in RPMI-1640 in the Absence and Presence of Human Serum
}

\author{
Renátó Kovács ${ }^{1}\left(\mathbb{D}\right.$, Zoltán Tóth ${ }^{1,2}$, Jeffrey B. Locke ${ }^{3}$, Lajos Forgács ${ }^{1,2}$, Gábor Kardos ${ }^{1}$, Fruzsina Nagy ${ }^{1,2}$, \\ Andrew M. Borman 4,5 and László Majoros ${ }^{1, *}$
}

check for updates

Citation: Kovács, R.; Tóth, Z.; Locke, J.B.; Forgács, L.; Kardos, G.; Nagy, F.; Borman, A.M.; Majoros, L. Comparison of In Vitro Killing Activity of Rezafungin, Anidulafungin, Caspofungin, and Micafungin against Four Candida auris Clades in RPMI-1640 in the Absence and Presence of Human Serum. Microorganisms 2021, 9, 863. https://doi.org/10.3390/ microorganisms 9040863

Academic Editor: Guillermo Quindos

Received: 23 March 2021

Accepted: 15 April 2021

Published: 16 April 2021

Publisher's Note: MDPI stays neutral with regard to jurisdictional claims in published maps and institutional affiliations.

Copyright: (c) 2021 by the authors. Licensee MDPI, Basel, Switzerland. This article is an open access article distributed under the terms and conditions of the Creative Commons Attribution (CC BY) license (https:// creativecommons.org/licenses/by/ $4.0 /)$.
1 Department of Medical Microbiology, Faculty of Medicine, University of Debrecen, 4032 Debrecen, Hungary; kovacs.renato@med.unideb.hu (R.K.); toth.zoltan@med.unideb.hu (Z.T.); forgacs.lajos.89@gmail.com (L.F.); kg@med.unideb.hu (G.K.); nagyfruzsina0429@gmail.com (F.N.)

2 Doctoral School of Pharmaceutical Sciences, University of Debrecen, 4032 Debrecen, Hungary

3 Cidara Therapeutics, Inc., 6310 Nancy Ridge Dr., Suite 101, San Diego, CA 92121, USA; jlocke@cidara.com

4 UK National Mycology Reference Laboratory, Public Health England, Science Quarter, Southmead Hospital, Bristol BS10 5NB, UK; andy.borman@nbt.nhs.uk

5 Medical Research Council Centre for Medical Mycology (MRC CMM), University of Exeter, Exeter EX4 4QD, UK

* Correspondence: major@med.unideb.hu; Tel.: +36-52-255-425; Fax: +36-52-255-424

Abstract: Candida auris is an emerging and frequently multidrug-resistant pathogen against which the echinocandins are the preferred therapeutic option. We compared killing activities of anidulafungin, caspofungin, micafungin, and rezafungin against 13 isolates representing four $C$. auris clades (South Asian $n=3$; East Asian $n=3$; South African $n=3$; South American $n=4$, of which two were of environmental origin). Minimum inhibitory concentration MICs and killing kinetics in RPMI1640 and RPMI-1640 plus 50\% serum (50\% serum) were determined. The four echinocandins were never fungicidal and induced large aggregates in RPMI-1640 and, less markedly, in 50\% serum. Colony forming unit CFU decreases were found more consistently in 50\% serum than in RPMI-1640. Isolates from the East Asian clade were killed at $\geq 1-\geq 4 \mathrm{mg} / \mathrm{L}$ with all echinocandins regardless of media. Anidulafungin and micafungin produced killing at peak drug serum concentration $(8 \mathrm{mg} / \mathrm{L})$ against environmental but not clinical isolates from the South American and the South African clades. Micafungin at $\geq 8 \mathrm{mg} / \mathrm{L}$ but not anidulafungin produced CFU decreases against the South Asian clade as well. In $50 \%$ serum, rezafungin at $\geq 1-\geq 8 \mathrm{mg} / \mathrm{L}$ produced killing against all four clades. The next generation echinocandin, rezafungin, showed the same or better activity at clinically attainable trough concentration regardless of media, compared with anidulafungin, caspofungin, and micafungin against all four tested $C$. auris clades.

Keywords: Candida auris; rezafungin; echinocandin; killing rate; time kill; serum

\section{Introduction}

Candida auris is an emerging fungal pathogen causing asymptomatic yet ubiquitous colonization and life-threatening invasive infections among critically ill patients in intensive care units [1]. Based on whole-genome sequence data of global clinical isolates, five phylogenetically distinct clades (South Asian, East Asian, South African, South American, and Iranian) were discovered to have emerged simultaneously on different continents [2,3]. C. auris lineages differ in their microscopic self-aggregating ability, growth in the presence of actidione, and ability to produce pseudomycelium on Dalmau cultures [4,5]; furthermore, significant clade-specific differences in virulence between South Asian, East Asian, South African, and South American lineages were detected using invertebrate and neutropenic murine models $[4,6,7]$. This pathogen is almost always multidrug resistant, leaving 
echinocandins (anidulafungin, caspofungin, and micafungin) as the current first-line therapeutic option for the treatment of $C$ auris infections $[1,8,9]$.

The echinocandins act as noncompetitive inhibitors of the $\beta-1,3-D-$ glucan synthase, which is responsible for the biosynthesis of $\beta-1,3-\mathrm{D}$-glucan, an essential structural component of fungal cell walls. In vitro, echinocandins show fungicidal or fungistatic activity against Candida species, including azole-resistant C. krusei or C. glabrata isolates [10]. The efficacy of echinocandins correlates with the ratios of area under the concentration curve per minimum inhibitory concentration (AUC/MIC) or serum peak drug concentration per minimum inhibitory concentration $\left(\mathrm{C}_{\max } / \mathrm{MIC}\right)$. All echinocandins are highly protein bound $(\geq 97.5 \%)$, which decreases the free thus active drug concentration, which may affect standard susceptibility testing in vitro. Echinocandin resistance is rare and is associated with mutations in FKS genes, which encode the catalytic subunit of $\beta$-1,3-D-glucan synthase [10].

Rezafungin is a next-generation echinocandin with distinctive pharmacokinetics, including prolonged half-life ( $>130 \mathrm{~h}$ ) and once-weekly intravenous administration [11-13]. An initial dose of $400 \mathrm{mg}$ rezafungin produces $22.7 \mathrm{mg} / \mathrm{L} \mathrm{C}$ max and $1160 \mathrm{mg} \cdot \mathrm{h} / \mathrm{L}$ AUC values in the first week, pharmacokinetic parameters, which are 2.95- to 3.24-fold and 1.4to 1.9-fold higher, respectively, compared to $C_{\max }$ and AUC values produced by standard daily doses of anidulafungin, caspofungin, and micafungin [10,12]. In vitro, rezafungin activity is comparable to that of the three approved echinocandins against common and uncommon Candida species, including C. auris $[14,15]$. However, data on killing kinetics against different $C$. auris clades are absent. Therefore, we compared the killing activities of anidulafungin, caspofungin, micafungin, and rezafungin against the four prevalent $C$. auris clades (South Asian, East Asian, South African, and South American). Since rezafungin, similar to other echinocandins, is highly protein bound with $0.2-3 \%$ free drug, killing activity was determined in standard RPMI-1640 medium and in RPMI-1640 containing $50 \%$ human serum (50\% serum) [16].

\section{Materials and Methods}

2.1. Isolates

All isolates, representing four C. auris clades (South Asian $n=3$, East Asian $n=3$, South African $n=3$, South American $n=4$ ), derived from our previous study (Table 1) [6]. Strains were stored at $-70{ }^{\circ} \mathrm{C}$. Two days before the in vivo experiments, isolates were subcultured using Sabouraud agar and screened on CHROMagar Candida (Becton Dickinson) to ensure the purity of Candida isolates.

\subsection{Antifungal Susceptibility Testing}

Rezafungin pure powder was provided by Cidara Therapeutics (San Diego, CA, USA). Caspofungin, micafungin, and anidulafungin were obtained from Molcan Corporation (Richmond Hill, ON, Canada). Minimum inhibitory concentration (MIC) values were determined simultaneously using the standard Clinical Laboratory Standars Institute (CLSI) broth macrodilution method in RPMI-1640 and in RPMI-1640 supplemented with $50 \%$ serum (human serum from a human male, type $A B$, not heat inactivated, Sigma, Budapest, Hungary) [16-19]. Antifungals were dissolved in 100\% DMSO and diluted further in RPMI-1640 to final concentrations between $0.015-8 \mathrm{mg} / \mathrm{L}$. MICs were read visually after $24 \mathrm{~h}$ using the partial inhibition criterion. For categorization, tentative MIC breakpoints as suggested by the Centers for Disease Control and Prevention were used: $\leq 4 \mathrm{mg} / \mathrm{L}$ for anidulafungin, $\leq 2 \mathrm{mg} / \mathrm{L}$ for caspofungin, and $\leq 4 \mathrm{mg} / \mathrm{L}$ for micafungin [9].

\subsection{Time-Kill Studies}

The killing activity was determined in RPMI-1640 with and without $50 \%$ human serum at concentrations $1-32 \mathrm{mg} / \mathrm{L}$ and at $0.25-32 \mathrm{mg} / \mathrm{L}$, respectively, in a final volume of $10 \mathrm{~mL}$ [19]. These echinocandin ranges were based on previous pharmacokinetic data with standard doses of currently approved echinocandins among intensive care 
unit patients [20-22] and Phase 1 data for rezafungin [12]. The starting inocula were $2.5-7 \times 10^{5} \mathrm{CFU} / \mathrm{mL}$. Aliquots of $100 \mathrm{~mL}$ were removed at $0,4,8,12$, and $24 \mathrm{~h}$, serially diluted ten-fold, plated $(4 \times 30 \mathrm{~mL})$ onto a single Sabouraud dextrose agar, and incubated at $35^{\circ} \mathrm{C}$ for $48 \mathrm{~h}$. All experiments were performed in both media twice [19].

Table 1. Minimum inhibitory concentration (MIC) values of rezafungin (RZF), anidulafungin (ANF), caspofungin (CSF), and micafungin (MCF) in RPMI-1640-/ + 50\% human serum against Candida auris isolates and type strain. MICs were determined three times using the Clinical Laboratory Standars Institute broth macrodilution method.

\begin{tabular}{|c|c|c|c|c|c|c|}
\hline \multirow{2}{*}{ Isolates Number } & \multirow{2}{*}{ Clade } & \multirow{2}{*}{ Body Site } & \multicolumn{4}{|c|}{ MIC Values in RPMI/RPMI + 50\% Serum $(\mathrm{mg} / \mathrm{L})$} \\
\hline & & & RZF & ANF & CSF & MCF \\
\hline 12 & South Asian & unknown & $0.03-0.06 / 0.5$ & $0.12 / 2$ & $1 / 0.5-1$ & $0.12-0.25 / 2$ \\
\hline 20 (NCPF 8985) & South Asian & Wound & $0.25 / 0.5$ & $0.25 / 1$ & $1 / 2$ & $0.25 / 2$ \\
\hline 27 (NCPF 89891) & South Asian & Pleural fluid & $0.12 / 1$ & $0.12 / 2$ & $0.5 / 2$ & $0.25 / 2$ \\
\hline $\begin{array}{c}\text { Type strain } \\
\text { (NCPF } \\
13029=\text { CBS } \\
10913)\end{array}$ & East Asian & External ear & $0.06 / 0.5$ & $0.03 / 0.5-1$ & $0.25 / 0.5$ & $0.12 / 2-4$ \\
\hline 15 (NCPF 8984) & East Asian & External ear & $0.03 / 0.25-0.5$ & $0.03-0.06 / 2$ & $0.5-1 / 1$ & $0.12 / 2$ \\
\hline 12372 (CBS 12372) & East Asian & Blood & $0.06 / 0.5$ & $0.03 / 0.5-1$ & $0.12 / 0.5$ & $0.03 / 2$ \\
\hline 204 & South African & Tracheostomy & $0.06 / 0.25-0.5$ & $0.03 / 2$ & $0.25 / 0.5-1$ & $0.12-0.25 / 2$ \\
\hline 2 (NCPF 8977) & South African & $\begin{array}{l}\text { Cerebrospinal } \\
\text { fluid }\end{array}$ & $0.12 / 1$ & $0.03 / 2$ & $0.5 / 1$ & $0.25 / 2-4$ \\
\hline 185 & South African & Blood & $0.12 / 1$ & $0.25 / 2$ & $0.25 / 1$ & $0.25 / 4$ \\
\hline $\mathrm{I}-24$ & $\begin{array}{l}\text { South American } \\
\text { (Israel) }\end{array}$ & Blood & $0.25 / 0.5$ & $0.06 / 4$ & $0.25 / 1$ & $0.12 / 4$ \\
\hline $\mathrm{I}-172$ & $\begin{array}{l}\text { South American } \\
\text { (Israel) }\end{array}$ & Blood & $0.25 / 0.5$ & $0.06 / 2$ & $0.25 / 1$ & $0.12 / 2$ \\
\hline $\begin{array}{c}13108 \text { (CDC } \\
\text { B-13108) }\end{array}$ & $\begin{array}{l}\text { South American } \\
\text { (Colombia) }\end{array}$ & $\begin{array}{c}\text { Hospital } \\
\text { environment }\end{array}$ & $0.12 / 0.5$ & $0.06 / 2$ & $0.25 / 1$ & $0.12 / 4$ \\
\hline $\begin{array}{c}16565 \text { (CDC } \\
\text { B-16565) }\end{array}$ & $\begin{array}{l}\text { South American } \\
\text { (Colombia) }\end{array}$ & $\begin{array}{c}\text { Hospital } \\
\text { environment }\end{array}$ & $0.12 / 0.25$ & $0.015 / 0.5$ & $0.25 / 0.5$ & $0.06 / 2$ \\
\hline
\end{tabular}

Killing kinetics at the tested concentrations were analyzed in both media (RPMI and RPMI-1640 plus 50\% serum), as described previously. Positive killing rate $(k)$ values indicate killing, and negative $k$ values indicate growth. Since echinocandins never produced a fungicidal effect against $C$. auris isolates, only the mean times to achieve a $50 \%$ reduction of the starting inoculum $\left(\mathrm{T}_{50}=0.30103 / k\right)$ were calculated from the $k$ values for each isolate and concentration in both media [19].

One-way ANOVA with Tukey's post-testing was used to determine significant differences in killing kinetics among isolates and concentrations in either RPMI-1640 or 50\% serum. T-test (with Welch's correction, where appropriate) was used for the same echinocandin concentrations in RPMI-1640 and 50\% serum to determine significant differences in killing kinetics in the different media [19].

\subsection{Phase-Contrast Microscopy}

Echinocandin-induced morphological alterations were examined at 1 and $16 \mathrm{mg} / \mathrm{L}$ with two isolates from each clade (isolates 12, 27, 15, 12,372, 204, 2, 13,108, and I-172) with all echinocandins after $24 \mathrm{~h}$ of incubation at $37^{\circ} \mathrm{C}$ in both media, with a Zeiss Axioskop 2 mot microscope coupled with a Zeiss Axiocam HRc camera using the phase-contrast technique. Image acquisition was performed, using Zeiss Axiovision 4.8.2 software. The total volume examined was $10 \mu \mathrm{L}$. 


\section{Results}

\subsection{MIC Values of the Echinocandins against $C$. auris}

In RPMI-1640, MICs of anidulafungin, caspofungin, and micafungin were not higher than the suggested tentative breakpoints for $C$. auris regardless of clades (Table 1) [9]. Rezafungin showed comparable MICs with the approved echinocandins. In 50\% serum, MICs were higher with all four drugs, with the lowest range $(0.25-1 \mathrm{mg} / \mathrm{L})$ observed for rezafungin, which may be related to relatively lower protein binding [10,16]. MIC increases in serum were the lowest (1- to 8-fold) and highest (8- to 64-fold) with caspofungin and anidulafungin, respectively.

\subsection{Time-Kill Studies}

All four echinocandins, regardless of clade and medium (RPMI-1640+/-50\% serum), were fungistatic (less than $99.9 \%$ reduction in viable cell count, compared to the starting inoculum) against $C$. auris isolates. Inhibition of isolates was frequently found only in the first 8-12 $\mathrm{h}$ with prominent regrowth after $24 \mathrm{~h}$. Time-kill plots of isolate I-172 from the South American clade are observed in both media in Figure 1.
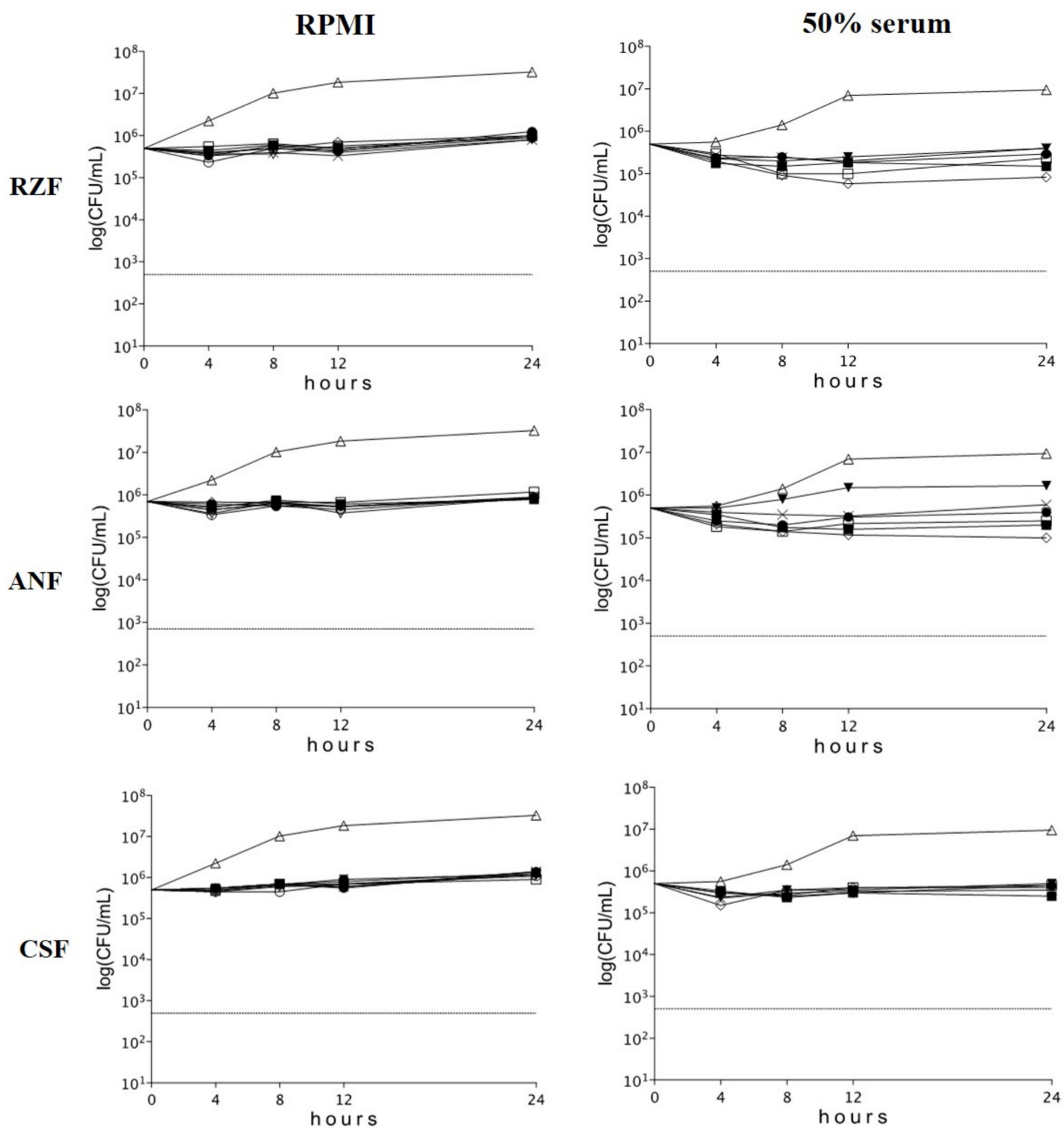

Figure 1. Cont. 


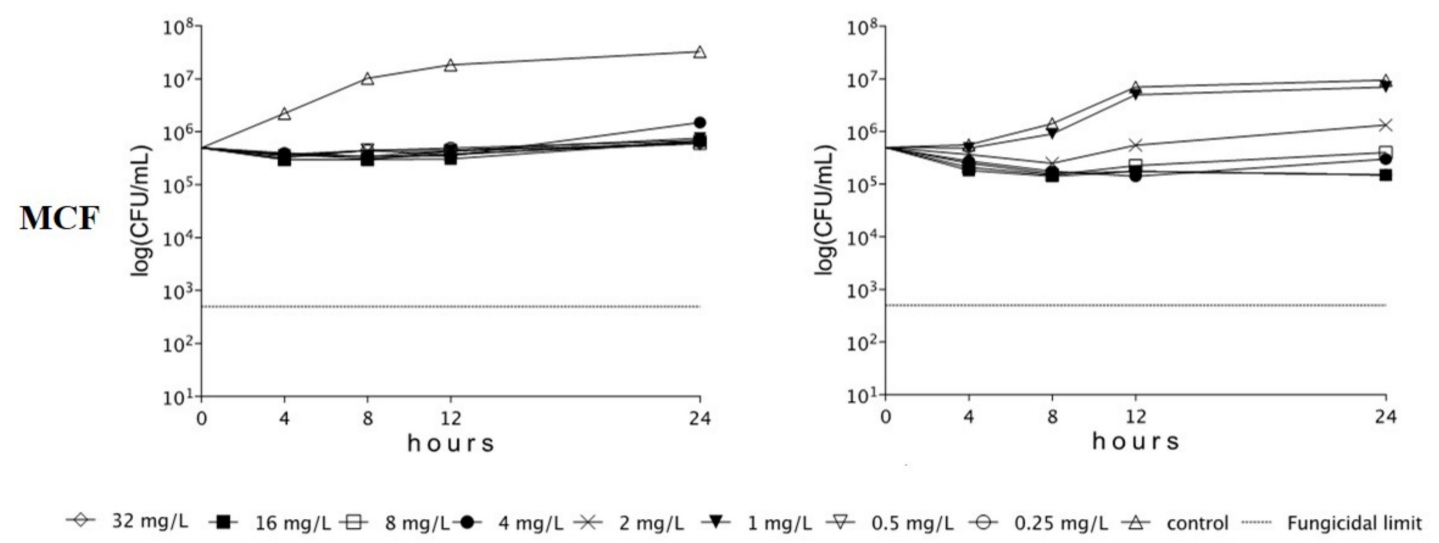

Figure 1. Time-kill plots of rezafungin (RZF), anidulafungin (ANF), caspofungin (CSF), and micafungin (MCF) in RPMI-1640 (RPMI) and RPMI-1640 plus 50\% human serum (50\% serum) against Candida auris isolate I-172, belonging to the South American clade.

\subsection{South Asian Clade}

In RPMI-1640, the mean killing rates were concentration dependent at $0.25-32 \mathrm{mg} / \mathrm{L}$ for rezafungin, anidulafungin, and micafungin. However, only rezafungin at $4-32 \mathrm{mg} / \mathrm{L}$ and anidulafungin at $16-32 \mathrm{mg} / \mathrm{L}$ produced uniformly positive $k$ values against the three isolates (Table 2 and Figure 2). Rezafungin $k$ values, in the case of isolate 27 at $0.25-2 \mathrm{mg} / \mathrm{L}$, were higher $(0.22-0.271 / \mathrm{h})$ than at $4-32 \mathrm{mg} / \mathrm{L}(0.10-0.141 / \mathrm{h})$ (mini-paradoxical effect).

Table 2. Average time (hours) to reach $50 \%$ growth reduction $\left(\mathrm{T}_{50}=0.30103 / k\right)$ from the starting inocula at different rezafungin (RZF), anidulafungin (ANF), caspofungin (CSF), and micafungin (MCF) concentrations in RPMI- $1640-/+50 \%$ human serum against 4 Candida auris clades. Data of the type strain (NCPF $13029=$ CBS 10913) were not included in the data of the East Asian clade. Data in bold indicate that $k$ values were positive (indicating killing) for all isolates within a clade, all other data (nonbold) indicate that one or two isolates never reached 50\% growth reduction; the datum represents the average of those isolates in which the $50 \%$ growth reduction was reached. NA: $50 \%$ growth inhibition not achieved; ND: not determined.

\begin{tabular}{|c|c|c|c|c|c|c|c|c|c|c|}
\hline \multirow{2}{*}{$50 \%$ Serum } & \multirow{2}{*}{ Clade } & \multirow{2}{*}{ Drug } & \multicolumn{8}{|c|}{ Time (Hours) } \\
\hline & & & $32 \mathrm{mg} / \mathrm{L}$ & $16 \mathrm{mg} / \mathrm{L}$ & $8 \mathrm{mg} / \mathrm{L}$ & $4 \mathrm{mg} / \mathrm{L}$ & $2 \mathrm{mg} / \mathrm{L}$ & $1 \mathrm{mg} / \mathrm{L}$ & $0.5 \mathrm{mg} / \mathrm{L}$ & $0.25 \mathrm{mg} / \mathrm{L}$ \\
\hline \multirow{20}{*}{$(-)$} & \multirow{4}{*}{$\begin{array}{l}\text { South Asian } \\
\text { (3 isolates) }\end{array}$} & RZF & 1.77 & 1.77 & 3 & 3.75 & 1.36 & 1.09 & 1.23 & 1.19 \\
\hline & & ANF & 3.1 & 3.4 & 3.4 & 2.14 & 2.14 & 3.51 & 1.22 & 1.82 \\
\hline & & MCF & 1.54 & 1.62 & 1.56 & 1.27 & 1.58 & 0.75 & 2 & 2.06 \\
\hline & & CSF & NA & NA & NA & NA & NA & NA & NA & NA \\
\hline & \multirow{4}{*}{$\begin{array}{l}\text { East Asian } \\
\text { ( } 2 \text { isolates) }\end{array}$} & RZF & 0.67 & 0.81 & 10 & 23 & NA & 15.5 & 15.3 & 16.4 \\
\hline & & ANF & 1 & 1.07 & 1.25 & 30.1 & 24.6 & 19.3 & 21.6 & 13.8 \\
\hline & & MCF & 1.3 & 6.95 & 1.56 & 13.6 & 18 & 31.6 & 69.7 & NA \\
\hline & & CSF & 1.38 & NA & NA & NA & NA & NA & NA & NA \\
\hline & \multirow{4}{*}{$\begin{array}{l}\text { South African } \\
\text { (3 isolates) }\end{array}$} & RZF & NA & NA & NA & NA & NA & NA & NA & NA \\
\hline & & ANF & NA & NA & NA & NA & NA & NA & NA & NA \\
\hline & & $\mathrm{MCF}$ & NA & NA & NA & NA & NA & NA & NA & NA \\
\hline & & CSF & NA & NA & NA & NA & NA & NA & NA & NA \\
\hline & \multirow{4}{*}{$\begin{array}{l}\text { South American } \\
\text { (2 isolates from Israel) }\end{array}$} & RZF & NA & NA & NA & NA & NA & NA & NA & NA \\
\hline & & ANF & NA & NA & NA & NA & NA & NA & NA & NA \\
\hline & & MCF & NA & NA & NA & NA & NA & NA & NA & NA \\
\hline & & CSF & NA & NA & NA & NA & NA & NA & NA & NA \\
\hline & \multirow{4}{*}{$\begin{array}{l}\text { South American } \\
\text { ( } 2 \text { isolates from } \\
\text { Colombia) }\end{array}$} & RZF & 5 & 2.7 & 1.43 & 1.36 & 1.11 & 1.11 & 0.88 & 1.58 \\
\hline & & ANF & 1.58 & 1.42 & 1.15 & 1.08 & 1.5 & 0.91 & 0.71 & 1.25 \\
\hline & & MCF & 2.14 & 2.07 & 1.58 & 1.43 & 1.36 & 1.15 & 1.3 & 0.91 \\
\hline & & CSF & NA & NA & NA & 100.3 & 29.4 & 15 & 6 & 3.75 \\
\hline
\end{tabular}


Table 2. Cont

\begin{tabular}{|c|c|c|c|c|c|c|c|c|c|c|}
\hline \multirow{2}{*}{$50 \%$ Serum } & \multirow{2}{*}{ Clade } & \multirow{2}{*}{ Drug } & \multicolumn{8}{|c|}{ Time (Hours) } \\
\hline & & & $32 \mathrm{mg} / \mathrm{L}$ & $16 \mathrm{mg} / \mathrm{L}$ & $8 \mathrm{mg} / \mathrm{L}$ & $4 \mathrm{mg} / \mathrm{L}$ & $2 \mathrm{mg} / \mathrm{L}$ & $1 \mathrm{mg} / \mathrm{L}$ & $0.5 \mathrm{mg} / \mathrm{L}$ & $0.25 \mathrm{mg} / \mathrm{L}$ \\
\hline \multirow{20}{*}{$(+)$} & \multirow{4}{*}{$\begin{array}{l}\text { South Asian } \\
\text { (3 isolates) }\end{array}$} & RZF & 1.77 & 3.34 & 6.69 & 15.1 & 75.3 & NA & ND & ND \\
\hline & & ANF & 1.5 & 3.75 & 7.5 & 7.5 & NA & NA & ND & ND \\
\hline & & $\mathrm{MCF}$ & 1.58 & 2.73 & 4.29 & 4.29 & NA & NA & ND & ND \\
\hline & & CSF & 3.75 & 75 & NA & NA & NA & NA & ND & ND \\
\hline & \multirow{4}{*}{$\begin{array}{l}\text { East Asian } \\
(2 \text { isolates })\end{array}$} & RZF & 0.71 & 1.08 & 1.11 & 1.2 & 1.25 & 1.3 & ND & ND \\
\hline & & ANF & 0.77 & 0.77 & 0.91 & 0.94 & 1.67 & NA & ND & ND \\
\hline & & $\mathrm{MCF}$ & 0.64 & 0.97 & 1 & 1.07 & 0.97 & NA & ND & ND \\
\hline & & CSF & 0.97 & 1.58 & 1.97 & 1.77 & 1.97 & 1.88 & ND & ND \\
\hline & \multirow{4}{*}{$\begin{array}{l}\text { South African } \\
\text { (3 isolates) }\end{array}$} & RZF & 3.42 & 3.81 & 4.3 & 1.85 & 1.88 & 100 & ND & ND \\
\hline & & ANF & 2.04 & 3.01 & 2.08 & 2.48 & 6.53 & NA & ND & ND \\
\hline & & $\mathrm{MCF}$ & 2.73 & 3.75 & 4.29 & 7.3 & NA & NA & ND & ND \\
\hline & & CSF & 17.2 & 43.8 & NA & NA & 21.1 & NA & ND & ND \\
\hline & \multirow{4}{*}{$\begin{array}{l}\text { South American } \\
\text { ( } 2 \text { isolates from Israel) }\end{array}$} & RZF & 3.76 & 7.07 & 10.03 & 12.57 & 38.2 & 39.2 & ND & ND \\
\hline & & ANF & 5.38 & 7.17 & 7.61 & 80.1 & NA & NA & ND & ND \\
\hline & & $\mathrm{MCF}$ & 7 & 4.27 & 24.6 & 9.56 & NA & NA & ND & ND \\
\hline & & CSF & NA & 17.3 & NA & NA & 67.3 & NA & ND & ND \\
\hline & \multirow{4}{*}{$\begin{array}{l}\text { South American } \\
\text { (2 isolates from } \\
\text { Colombia) }\end{array}$} & RZF & 1.37 & 1.63 & 2.14 & 2.17 & 2.57 & 3.64 & ND & ND \\
\hline & & ANF & 1.39 & 1.92 & 2.57 & 1.41 & 1.89 & 15.1 & ND & ND \\
\hline & & MCF & 2.04 & 1.96 & 3.08 & 1.76 & 45.5 & NA & ND & ND \\
\hline & & CSF & 1.81 & 2.15 & 1.95 & 1.78 & 2.01 & 1.74 & ND & ND \\
\hline
\end{tabular}
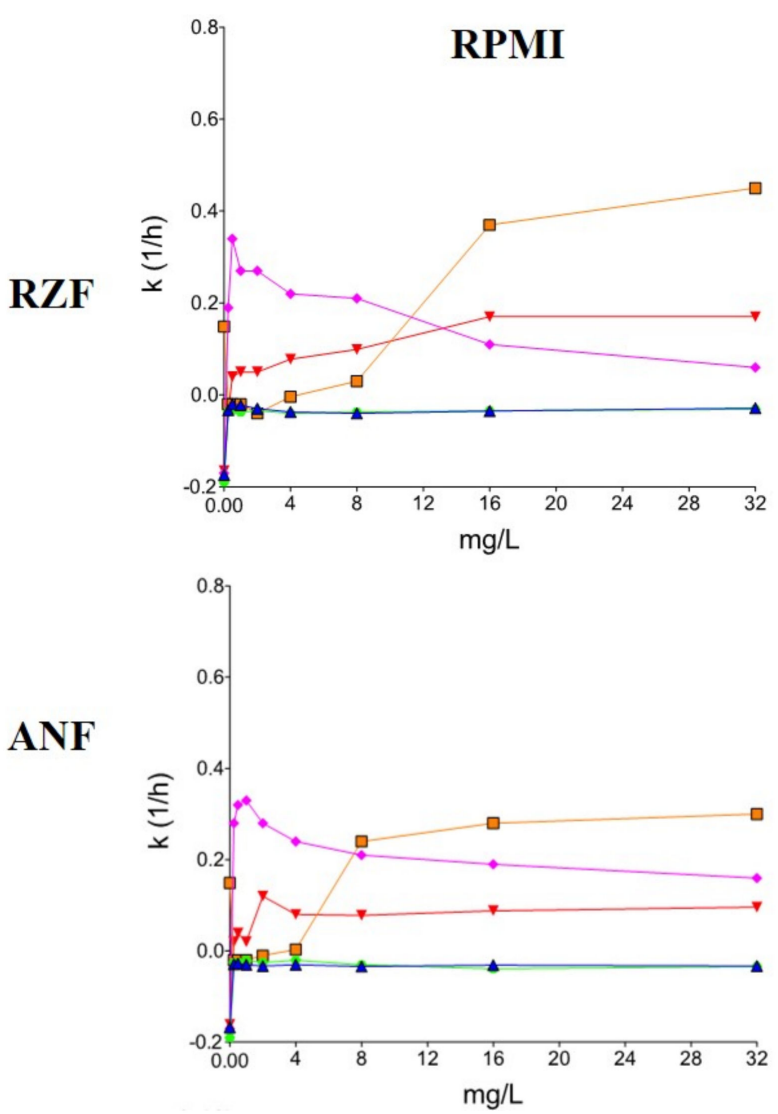
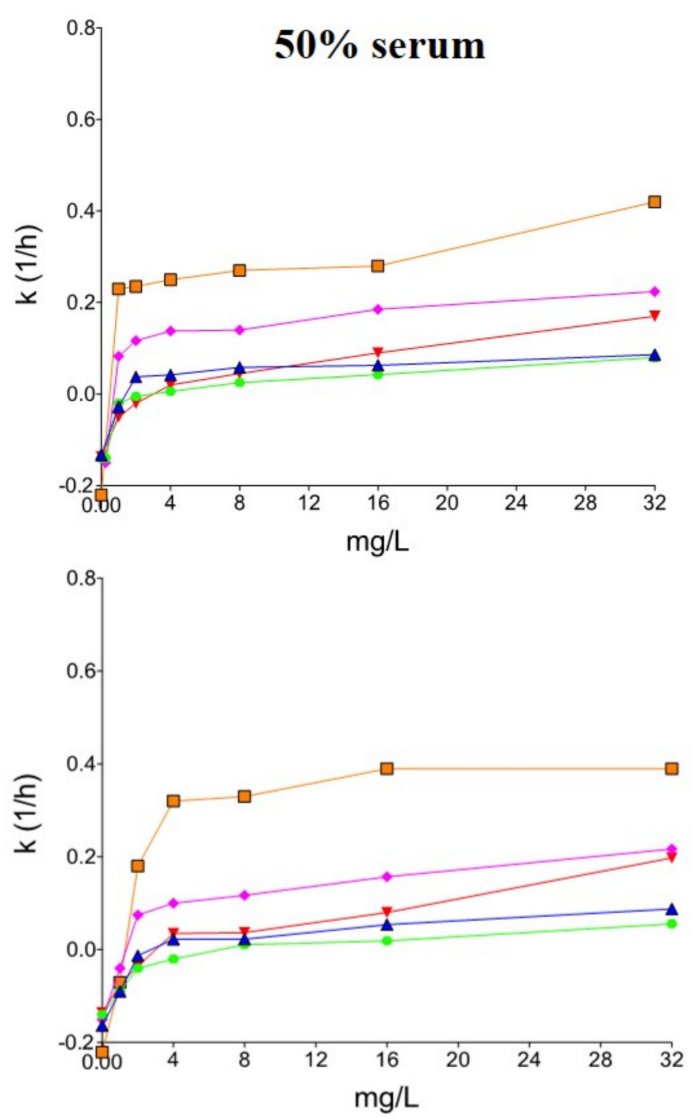

Figure 2. Cont. 

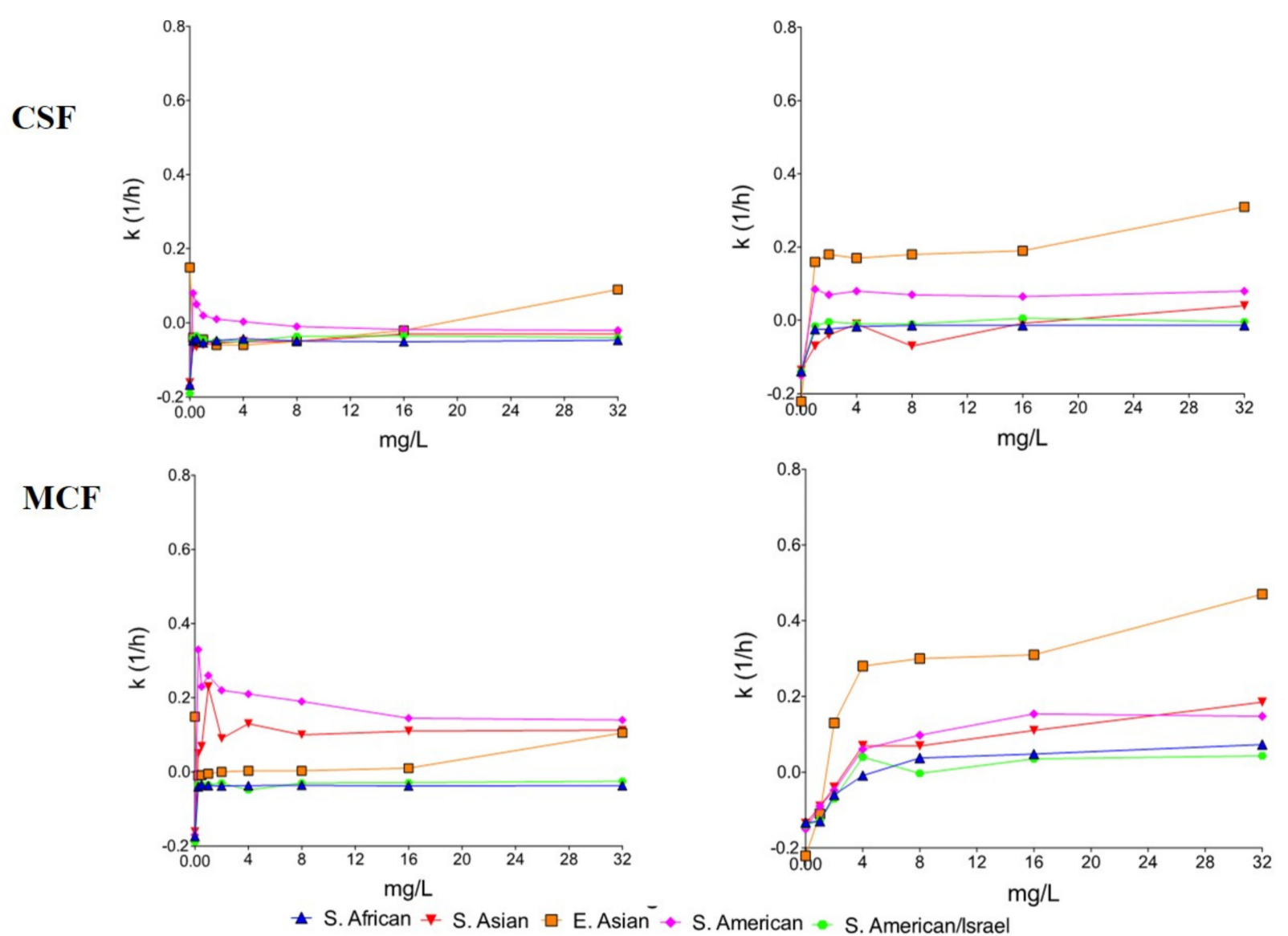

Figure 2. Mean killing rate values of rezafungin (RZF), anidulafungin (ANF), caspofungin (CSF), and micafungin (MCF) in RPMI-1640 (RPMI) and RPMI-1640 plus 50\% human serum (50\% serum) against 4 Candida auris clades. Data of the type strain (NCPF 13029 = CBS 10913) were not included in the East Asian clade. Positive and negative $k$ values indicate the decrease and increase, respectively, in viable cell numbers. Error bars were omitted for better visualization of the graphics.

In $50 \%$ serum, rezafungin, anidulafungin, and micafungin at $4-32 \mathrm{mg} / \mathrm{L}(\geq 2-4 \times \mathrm{MIC})$ produced concentration-dependent killing activity without regrowth $(p>0.05$ comparing these three echinocandins). In 50\% serum, the mean anidulafungin $k$ value at $32 \mathrm{mg} / \mathrm{L}$ $(k=0.201 / \mathrm{h})$ was higher than in RPMI-1640 $(k=0.101 / \mathrm{h})(p<0.05)$. Caspofungin $k$ values were positive against all three isolates only at $32 \mathrm{mg} / \mathrm{L}$ (mean $k$ value was $0.041 / \mathrm{h}$.) (Table 2 and Figure 2).

\subsection{East Asian Clade}

In RPMI-1640, rezafungin, anidulafungin, and caspofungin at $0.25-32 \mathrm{mg} / \mathrm{L}$ showed concentration-dependent killing activity against the $C$. auris type strain, producing colony forming unit (CFU) decreases close to the fungicidal limit at $32 \mathrm{mg} / \mathrm{L}$ (2.6-2.9 log decrease) (data not shown). Micafungin showed greater killing activity at $0.25-2 \mathrm{mg} / \mathrm{L}$ ( $k$ value ranges were $0.21-0.251 / \mathrm{h}$ ), compared to $4-32 \mathrm{mg} / \mathrm{L}$ ( $k$ values were $0.11-0.141 / \mathrm{h}$ ) (miniparadoxical growth). In 50\% serum, killing activity of rezafungin and caspofungin was concentration dependent at 1-32 mg/L. Micafungin and anidulafungin generated positive $k$ values at $\geq 4 \mathrm{mg} / \mathrm{L}$ and $\geq 2 \mathrm{mg} / \mathrm{L}$, respectively.

For the remaining two isolates in RPMI-1640, the mean $k$ values at lower concentrations $(0.25-4 \mathrm{mg} / \mathrm{L})$ were less consistently positive than in 50\% serum; in RPMI-1640, positive $k$ values were noticed against both isolates only with rezafungin and anidulafungin at $8-32 \mathrm{mg} / \mathrm{L}$. In contrast, in 50\% serum, rezafungin and caspofungin at $1-16 \mathrm{mg} / \mathrm{L}$, anidulafungin at $2-32 \mathrm{mg} / \mathrm{L}$, and micafungin at $4-32 \mathrm{mg} / \mathrm{L}$ produced concentration-independent killing activity against the two isolates (Table 2 and Figure 2). 


\subsection{South African Clade}

In RPMI-1640, South African isolates were inhibited but never killed by either of the four echinocandins, even at 256-1024× MIC. The highest CFU decreases $(-0.4 \log )$ was noticed with $1 \mathrm{mg} / \mathrm{L}$ anidulafungin against isolate 185 after $12 \mathrm{~h}$ (Figure 2).

In 50\% serum, the killing activity of echinocandins increased against all isolates. However, against all isolates, only rezafungin produced positive $k$ values (mean $k$ values at $8-32 \mathrm{mg} / \mathrm{L}$ were $0.07-0.09 \mathrm{1} / \mathrm{h}$ ) (Table 2 and Figure 2).

\subsection{South American Clade}

The isolates of the different origin in this clade behaved differently, i.e., bloodstream isolates from Israel showed a markedly different response to echinocandins from the behavior of Colombian environmental isolates. Israeli isolates were similar to the South African clade; in RPMI-1640, $k$ values were always negative (no killing) at all tested concentrations (Figure 2). In 50\% serum, rezafungin at $8-32 \mathrm{mg} / \mathrm{L}$ and anidulafungin and micafungin at $32 \mathrm{mg} / \mathrm{L}$ showed positive $k$ values against both isolates (mean $k$ values were $0.03-0.08,0.06$, and $0.041 / \mathrm{h}$, respectively) (Table 2 and Figure 2). Caspofungin always produced negative $k$ values with the two isolates at all tested concentrations.

Against the hospital environmental isolates from Colombia (isolates 13,108 and 16,565), all four echinocandins showed significantly greater killing at lower, than at higher, concentrations (mini-paradoxical effect) in RPMI-1640 (Table 2 and Figure 2). In 50\% serum, rezafungin, anidulafungin, and micafungin showed concentration-dependent killing, while caspofungin showed concentration-independent killing against the two isolates. In serum, rezafungin produced positive $k$ values even at $\geq 1 \mathrm{mg} / \mathrm{L}$ against both isolates (Figure 2).

\subsection{Phase-Contrast Microscopy}

Echinocandin-treated C. auris cells in RPMI-1640 frequently showed large (20-30 $\mu \mathrm{m}$ in diameter) aggregates with up to 100 cells, regardless of the clade. Neither the number nor the size of the aggregates was dependent on the echinocandin or its concentration (i.e., any echinocandin at 1 or $16 \mathrm{mg} / \mathrm{L}$ was able to induce large aggregates). In 50\% serum, the number and size of aggregates decreased significantly at both concentrations. Abnormal cell morphology and cell debris were typical after $24 \mathrm{~h}$ at $16 \mathrm{mg} / \mathrm{L}$ regardless of the echinocandin or clade (Figure 3). 


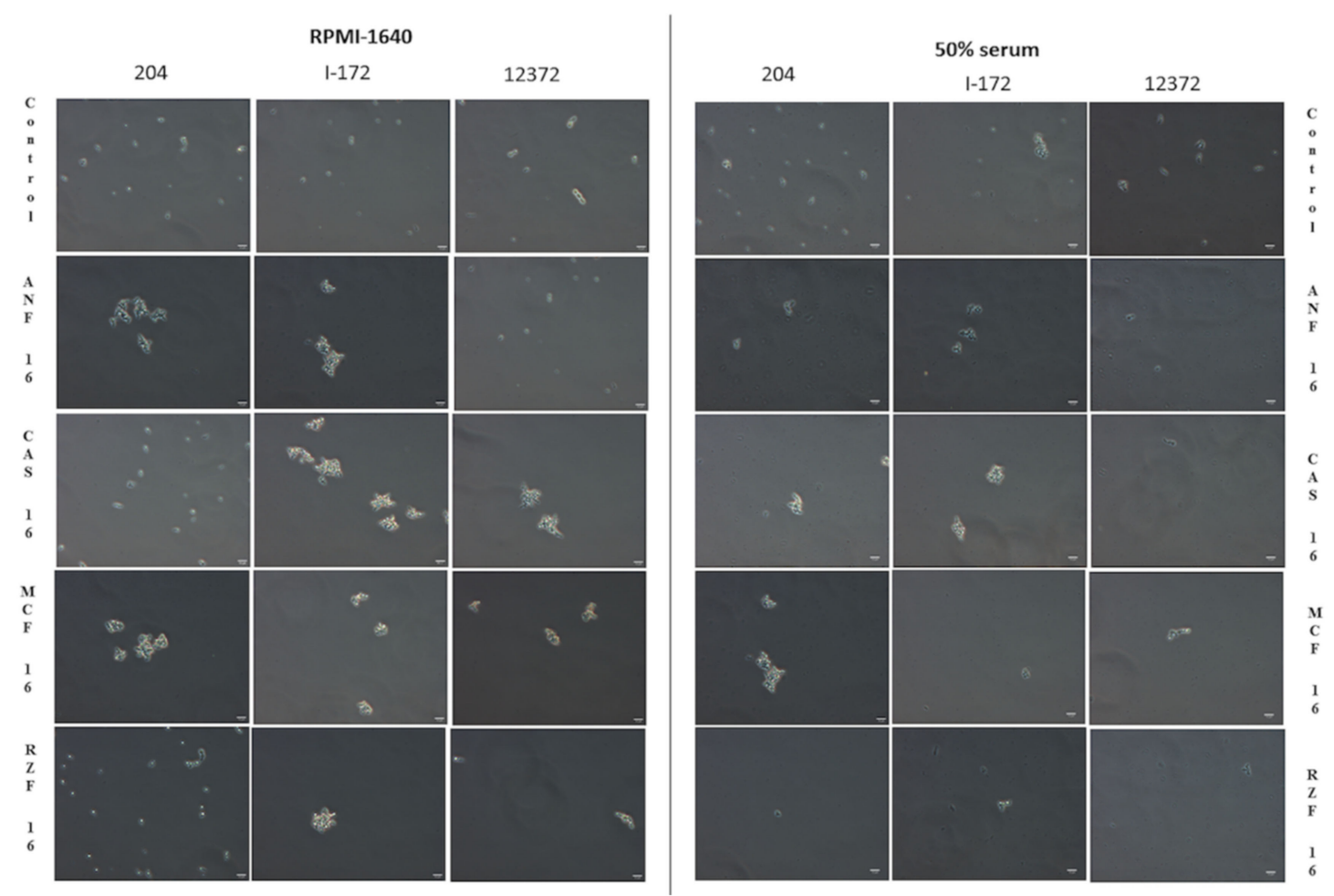

Figure 3. Phase-contrast microscopy images of untreated C. auris cells and cells treated with $16 \mathrm{mg} / \mathrm{L}$ rezafungin (RZF), $16 \mathrm{mg} / \mathrm{L}$ anidulafungin (ANF), $16 \mathrm{mg} / \mathrm{L}$ caspofungin (CSF), and $16 \mathrm{mg} / \mathrm{L}$ micafungin (MCF) in RPMI-1640 and RPMI-1640 plus 50\% human serum (50\% serum) against isolates 204 (South African clade), I-172 (South American clade) and 12,372 (East Asian clade). The micrograph was taken after $24 \mathrm{~h}$. In RPMI-1640, single and budding cells or short chains with small aggregates containing up to 3-6 cells were observed in controls. In 50\% serum, the appearance of the untreated isolates was similar, but small aggregates (up to 5-10 $\mu \mathrm{m}$ in diameter) were more frequently observed. In RPMI-1640, all four echinocandins induced large aggregates in the case of isolate I-172, and ANF and MCF in the case of isolate 204, while CSF and MCF in the case of isolate 12372. In 50\% serum, large aggregates could be observed only in cases of isolate 204 with CSF and MCF, and isolate I-172 with CSF. Abnormal cell morphology and cell debris were typical after $24 \mathrm{~h}$. Bar: $10 \mu \mathrm{m}$.

\section{Discussion}

This study demonstrated that in RPMI-1640, after $24 \mathrm{~h}$, the four echinocandins did not produce killing at $32 \mathrm{mg} / \mathrm{L}$ against the South African clade and isolates from Israel (South American clade). These isolates responded similarly to echinocandin exposure despite their significantly different virulence in a neutropenic murine model (South African isolates showed the least, while isolates from Israel the highest virulence) [6]. The two environmental isolates from the South American clade (Colombian isolates) were killed at $\geq 0.25 \mathrm{mg} / \mathrm{L}$, but mini-paradoxical growth was observed with all four echinocandins (Table 2). Against the two East Asian clinical isolates and South Asian clade, only rezafungin and anidulafungin (at $\geq 4-\geq 8 \mathrm{mg} / \mathrm{L}$ and $\geq 8-\geq 16 \mathrm{mg} / \mathrm{L}$, respectively) produced CFU decreases after $24 \mathrm{~h}$. However, all four echinocandins showed good killing activity against the C. auris type strain (NCPF 13029; CBS 10913).

Although MIC values increased in the presence of 50\% human serum, the killing activity of echinocandins increased against all clades at $\geq 2 \times$ MICs. In addition, in $50 \%$ serum, the occurrence of echinocandin-induced aggregates decreased significantly regardless of the clade (Figure 3). The killing activity of caspofungin was negligible, except against the East Asian clade and the two isolates from the South American clade. Anidulafungin and micafungin proved to be effective at peak concentration $(\sim 8 \mathrm{mg} / \mathrm{L})$ [20,22] against isolates from Colombia (South American clade) but not against isolates from Israel (South American clade) or the South African clade. The next-generation rezafungin showed the same or greater activity, at clinically attainable trough concentration $(4 \mathrm{mg} / \mathrm{L})$ regardless of 
media, than did anidulafungin and micafungin against the South Asian, East Asian, and South American (Colombian isolates) clades [12,20,22]. Moreover, a single 400-mg dose of rezafungin produces $\geq 8 \mathrm{mg} / \mathrm{L}$ serum levels even after $48-72 \mathrm{~h}$, which is capable of early enhanced killing and sustained therapeutic effect to maintain elimination of the fungus, including isolates from Israel (South American clade) and the South African clade, against which other echinocandins were less active [12].

Data on in vitro killing activity of echinocandins against C. auris are scant. Dudiuk et al. [23] determined the killing activity of caspofungin and anidulafungin in RPMI-1640 against 9 C. auris bloodstream isolates from Colombia. They reported slightly poorer killing, compared to our Colombian isolates (South American clade) (average $k$ value ranges for caspofungin and anidulafungin were between -0.006 to $+0.071 / \mathrm{h}$ and 0.005 to $0.071 / \mathrm{h}$, respectively). The lack of similar studies comparing rezafungin to the three approved echinocandins against different C. auris clades precludes comparative discussion of our results.

An important finding of our study is that, regardless of media, significant differences exist between in vitro killing by the currently approved echinocandins and by rezafungin against the four prevalent $C$. auris clades. MIC values do not reflect the weak inhibition without killing effect against some $C$. auris clades. One possible explanation is that echinocandin exposure, regardless of medium, clade, or isolate, induces large aggregates of C. auris, as demonstrated both in vitro and in vivo [4-6]. We have previously demonstrated that $C$. auris isolates from certain clades (particularly the South African clade) grow as large aggregates that cannot be physically disrupted by sonication, vortex mixing, or with detergents, and have proposed that this phenotype is due to failure to release daughter cells and complete abscission after cell division [4]. In addition, we have shown that this phenotype can also be reversibly induced in isolates of "nonaggregative" clades by exposure to low concentrations of those antifungal agents that affect cell wall and cell membrane integrity [5]. This phenotype is clearly due to defects in cytokinesis, which are constitutive in certain clades and drug inducible in others, rather than a physical clumping of individual cells, due to, for example, changes in cell surface properties [4,5]. This phenomenon has also been seen in vivo $[4,6]$. The aggregate formation may be a general survival strategy of $C$. auris, and yeast cells in the center of the aggregates could be protected from the antifungals and from immune effectors. Aggregate formation in vitro is necessary but not sufficient for $C$. auris cells to survive echinocandin exposure since a significant proportion of cells were killed in both media, even in the case of the more resistant isolates from Israel.

Another explanation for the weak fungistatic effect of echinocandins is that decreased amount of $\beta$-glucan induces a variety of stress adaptation pathways resulting in increased cell wall chitin $[10,24-26]$. Increased chitin content helps to stabilize the fungal cell wall, therefore mitigating the impact of echinocandins. Although this adaptive response does not lead to mutations in the FKS genes, the early adaptation to echinocandin exposure enhances survival of the drug effect and may allow the development of stable resistance mechanisms over time for surviving cells, possibly leading to clinical resistance in the long run [24-26]. The mini-paradoxical effect in RPMI-1640, which disappeared in the case of some isolates in the presence of serum, may represent such adaptation. The high protein binding decreases the free (i.e., active) echinocandin concentration as modeled by the presence of $50 \%$ serum, which is mirrored in elevated MICs [10]. Lower free drug levels induce less compensatory chitin synthesis; thus, higher $k$ values at higher concentrations with serum may be explained by the enhancement of the killing activities of echinocandins due to lower cell wall chitin, compared to RPMI-1640 (Figure 2) [10,26]. Although chitin content was not measured in our study, other authors found elevated chitin content in C. auris, compared to other species more susceptible to echinocandins [27]. Furthermore, fast increase of chitin content in response to caspofugin was reported with caspofungin-resistant but not caspofungin-susceptible C. auris isolates [28].

The in vivo efficacy of the four echinocandins was not determined in animal models using these isolates, which may be regarded as a limitation. However, other authors found that a human equivalent dose of micafungin ( $5 \mathrm{mg} / \mathrm{kg}$ daily) given early ( $2 \mathrm{~h}$ post-infection) 
was effective in decreasing the fungal kidney burdens in a neutropenic murine model against echinocandin-susceptible $C$. auris isolates from different countries [29]. Similar results were reported with a humanized dose of rezafungin (single $20 \mathrm{mg} / \mathrm{kg}$ ) against three C. auris isolates in a neutropenic murine model [30]. A combination of echinocandins with new triazoles may be a promising approach to improve the therapeutic efficacy further against $C$. auris [31,32].

An important implication of our findings, i.e., the currently used standard echinocandin regimens do not reliably produce serum concentrations high enough to eradicate C. auris from the bloodstream, may partially explain therapeutic failures among critically ill patients. This may also contribute to the continuous candidemia and unacceptably high mortality among SARS-CoV2-positive patients with $C$. auris infections in spite of treatment with standard doses of echinocandins $[9,29,33]$. The very high $C_{\max }$ and AUC values produced by once-weekly rezafungin in the blood and tissues and its excellent safety profile even at supratherapeutic doses (>400 mg weekly) in healthy subjects, together with promising findings in animal models [12,34], raise hopes that rezafungin may prove superior to the earlier echinocandins in treatment of life-threatening C. auris infections.

\section{Conclusions}

In vitro killing activities of echinocandins against C. auris were clade-, isolate-, and medium dependent. All four echinocandins induced large aggregates in RPMI-1640 and $50 \%$ serum, and killing in 50\% serum was more consistently positive than in RPMI-1640. The next-generation echinocandin, rezafungin, showed the same or greater activity at clinically attainable trough concentration regardless of media, compared with anidulafungin, caspofungin, and micafungin against the South Asian, East Asian, South American, and South African clades.

Author Contributions: Conceptualization, J.B.L., A.M.B., and L.M.; methodology, Z.T., L.F., and F.N.; laboratory analyses, R.K., G.K., and L.M.; writing-original draft preparation, R.K. and G.K.; supervision, L.M. All authors have read and agreed to the published version of the manuscript.

Funding: The study itself did not receive any direct funding. Renátó Kovács was supported by the EFOP-3.6.3-VEKOP-16-2017-00009 program, OTKA Bridging Fund, and FEMS Research and Training Grant (FEMS-GO-2019-502). Zoltán Tóth and Fruzsina Nagy were supported by the ÚNKP-19-3 and ÚNKP-20-3 New National Excellence Program of the Ministry for Innovation and Technology.

Institutional Review Board Statement: Not applicable.

Informed Consent Statement: Not applicable.

Data Availability Statement: Data are available from the corresponding author upon reasonable request.

Acknowledgments: Part of this work was presented at the Ninth Congress on Trends in Medical Mycology, 11-14 October 2019, Nice, France (P055).

Conflicts of Interest: L. Majoros has received conference travel grants from MSD, Cidara, Astellas, and Pfizer. J.B. Locke is an employee and shareholder of Cidara Therapeutics, Inc.

\section{References}

1. Sekyere, J.O. Candida Auris: A Systematic Review and Meta-Analysis of Current Updates on an Emerging Multidrug-Resistant Pathogen. Microbiol. Open 2018, 7, e00578. [CrossRef] [PubMed]

2. Lockhart, S.R.; Etienne, K.A.; Vallabhaneni, S.; Farooqi, J.; Chowdhary, A.; Govender, N.P.; Colombo, A.; Calvo, B.; Cuomo, C.A.; Desjardins, C.A. Simultaneous Emergence of Multidrug-Resistant Candida Auris on 3 Continents Confirmed by Whole-Genome Sequencing and Epidemiological Analyses. Clin. Infect. Dis. 2017, 64, 134-140. [CrossRef] [PubMed]

3. Chow, N.A.; de Groot, T.; Badali, H.; Abastabar, M.; Chiller, T.M.; Meis, J.F. Potential fifth Clade of Candida Auris, Iran, 2018. Emerg. Infect. Dis. 2019, 25, 1780-1781. [CrossRef] [PubMed]

4. Borman, A.M.; Szekely, A.; Johnson, E.M. Comparative Pathogenicity of United Kingdom Isolates of the Emerging Pathogen Candida auris and Other Key Pathogenic Candida Species. mSphere 2016, 1, e00189-16. [CrossRef] [PubMed]

5. Szekely, A.; Borman, A.M.; Johnson, E.M. Candida Auris Isolates of the Southern Asian and South African Lineages Exhibit Different Phenotypic and Antifungal Susceptibility Profiles In Vitro. J. Clin. Microbiol. 2019, 57, e02055-18. [CrossRef] 
6. $\quad$ Forgács, L.; Borman, A.; Prépost, E.; Tóth, Z.; Kardos, G.; Kovács, R.; Szekely, A.; Nagy, F.; Kovacs, I.; Majoros, L. Comparison of In Vivo Pathogenicity of Four Candida Auris Clades in a Neutropenic Bloodstream Infection Murine Model. Emerg. Microb. Infect. 2020, 9, 1160-1169. [CrossRef]

7. Hernando-Ortiz, A.; Mateo, E.; Perez-Rodriguez, A.; de Groot, P.W.J.; Quindós, G.; Eraso, E. Virulence of Candida Auris from Different Clinical Origins in Caenorhab-Ditis Elegans and Galleria Mellonella Host Models. Virulence 2021, 12, 1063-1075. [CrossRef]

8. Chowdhary, A.; Tarai, B.; Singh, A.; Sharma, A. Multidrug-Resistant Candida auris Infections in Critically Ill Coronavirus Disease Patients, India, April-July 2020. Emerg. Infect. Dis. 2020, 26, 2694-2696. [CrossRef]

9. CDC. Available online: https://www.cdc.gov/fungal/candida-auris/c-auris-antifungal.html (accessed on 22 March 2021).

10. Perlin, D.S. Echinocandin Resistance, Susceptibility Testing and Prophylaxis: Implications for Patient Management. Drugs 2014, 74, 1573-1585. [CrossRef]

11. Zhao, Y.; Perez, W.B.; Jiménez-Ortigosa, C.; Hough, G.; Locke, J.B.; Ong, V.; Bartizal, K.; Perlin, D.S. CD101: A Novel Long-Acting Echinocandin. Cell. Microbiol. 2016, 18, 1308-1316. [CrossRef]

12. Sandison, T.; Ong, V.; Lee, J.; Thye, D. Safety and Pharmacokinetics of CD101 IV, a Novel Echinocandin, in Healthy Adults. Antimicrob. Agents Chemother. 2017, 61, 01627-16. [CrossRef]

13. Sofjan, A.K.; Mitchell, A.; Shah, D.N.; Nguyen, T.; Sim, M.; Trojcak, A.; Beyda, N.D.; Garey, K.W. Rezafungin (CD101), a next-generation echinocandin: A systematic literature review and assessment of possible place in therapy. J. Glob. Antimicrob. Resist. 2018, 14, 58-64. [CrossRef]

14. Pfaller, M.A.; Carvalhaes, C.; Messer, S.A.; Rhomberg, P.R.; Castanheira, M. Activity of a Long-Acting Echinocandin, Rezafungin, and Comparator Antifungal Agents Tested against Contemporary Invasive Fungal Isolates (SENTRY Program, 2016 to 2018). Antimicrob. Agents Chemother. 2020, 64, 00099-20. [CrossRef]

15. Tóth, Z.; Forgács, L.; Locke, J.B.; Kardos, G.; Nagy, F.; Kovács, R.; Szekely, A.; Borman, A.; Majoros, L. In vitro activity of rezafungin against common and rare Candida species and Saccharomyces cerevisiae. J. Antimicrob. Chemother. 2019, 74, 3505-3510. [CrossRef]

16. Ong, V.; Hough, G.; Schlosser, M.; Bartizal, K.; Balkovec, J.M.; James, K.D.; Krishnan, B.R. Preclinical Evaluation of the Stability, Safety, and Efficacy of CD101, a Novel Echinocandin. Antimicrob. Agents Chemother. 2016, 60, 6872-6879. [CrossRef]

17. CLSI. Reference Method for Broth Dilution Antifungal Susceptibility Testing of Yeasts. Approved Standard-Third Edition M27-A3; Clinical and Laboratory Standard Institute: Wayne, PA, USA, 2008.

18. Clinical and Laboratory Standards Institute. Performance Standards for Antifungal Susceptibility Testing of Yeasts, 1st ed.; M60Ed1; CLSI: Wayne, PA, USA, 2017.

19. Domán, M.; Kovács, R.L.; Perlin, D.S.; Kardos, G.; Gesztelyi, R.; Juhász, B.; Bozó, A.; Majoros, L. Dose Escalation Studies with Caspofungin against Candida Glabrata. J. Med Microbiol. 2015, 64, 998-1007. [CrossRef]

20. Brüggemann, R.J.M.; Middel-Baars, V.; De Lange, D.W.; Colbers, A.; Girbes, A.R.J.; Pickkers, P.; Swart, E.L. Pharmacokinetics of Anidulafungin in Critically Ill Intensive Care Unit Patients with Suspected or Proven Invasive Fungal Infections. Antimicrob. Agents Chemother. 2016, 61, 01894-16. [CrossRef]

21. Muilwijk, E.W.; Schouten, J.A.; Van Leeuwen, H.J.; Van Zanten, A.R.H.; De Lange, D.W.; Colbers, A.; Verweij, P.E.; Burger, D.M.; Pickkers, P.; Brüggemann, R.J.M. Pharmacokinetics of caspofungin in ICU patients. J. Antimicrob. Chemother. 2014, 69, 3294-3299. [CrossRef]

22. Boonstra, J.M.; Van Der Elst, K.C.; Veringa, A.; Jongedijk, E.M.; Brüggemann, R.J.; Koster, R.A.; Kampinga, G.A.; Kosterink, J.G.; Van Der Werf, T.S.; Zijlstra, J.G.; et al. Pharmacokinetic Properties of Micafungin in Critically Ill Patients Diagnosed with Invasive Candidiasis. Antimicrob. Agents Chemother. 2017, 61, e01398-17. [CrossRef]

23. Dudiuk, C.; Berrio, I.; Leonardelli, F.; Morales-Lopez, S.; Theill, L.; Macedo, D.; Yesid-Rodriguez, J.; Salcedo, S.; Marin, A.; Gamarra, S.; et al. Antifungal activity and killing kinetics of anidulafungin, caspofungin and amphotericin B against Candida auris. J. Antimicrob. Chemother. 2019, 74, 2295-2302. [CrossRef]

24. Kordalewska, M.; Lee, A.; Park, S.; Berrio, I.; Chowdhary, A.; Zhao, Y.; Perlin, D.S. Understanding Echinocandin Resistance in the Emerging Pathogen Candida auris. Antimicrob. Agents Chemother. 2018, 62, e00238-18. [CrossRef]

25. Tóth, Z.; Forgács, L.; Kardos, T.; Kovács, R.; Locke, J.B.; Kardos, G.; Nagy, F.; Borman, A.M.; Adnan, A.; Majoros, L. Relative Frequency of Paradoxical Growth and Trailing Effect with Caspofungin, Micafungin, Anidulafungin, and the Novel Echinocandin Rezafungin against Candida Species. J. Fungi 2020, 6, 136. [CrossRef]

26. Perlin, D.S. Cell Wall-Modifying Antifungal Drugs. Curr. Top. Microbiol. Immunol. 2019, 425, 255-275. [CrossRef]

27. Navarro-Arias, M.J.; Hernández-Chávez, M.J.; Garcia-Carnero, L.C.; Amezcua-Hernández, D.G.; Lozoya-Pérez, N.E.; EstradaMata, E.; Martínez-Duncker, I.; Franco, B.; Mora-Montes, H.M. Differential Recognition of Candida Tropicalis, Candida Guilliermondii, Candida Krusei, and Candida Auris by Human Innate Immune Cells. Infect. Drug Resist. 2019, 12, 783-794. [CrossRef]

28. Sharma, D.; Paul, R.A.; Chakrabarti, A.; Bhattacharya, S.; Soman, R.; Shankarnarayan, S.A.; Chavan, D.; Singh, S.; Das, P.; Kaur, H.; et al. Caspofungin Resistance in Candia Auris due to Mutations in Fks1 with Adjunctive Role of Chitin and Key Cell Wall Stress Response Pathway Genes. bioRxiv 2020, 196600. [CrossRef]

29. Lepak, A.J.; Zhao, M.; Berkow, E.L.; Lockhart, S.R.; Andes, D.R. Pharmacodynamic Optimization for Treatment of Invasive Candida auris Infection. Antimicrob. Agents Chemother. 2017, 61, 00791-17. [CrossRef]

30. Lepak, A.J.; Zhao, M.; Andes, D.R. Pharmacodynamic Evaluation of Rezafungin (CD101) against Candida auris in the Neutropenic Mouse Invasive Candidiasis Model. Antimicrob. Agents Chemother. 2018, 62, 01572-18. [CrossRef]

31. Caballero, U.; Kim, S.; Eraso, E.; Quindós, G.; Vozmediano, V.; Schmidt, S.; Jauregizar, N. In Vitro Synergistic Interactions of Isavuconazole and Echinocandins against Candida auris. Antibiotics 2021, 10, 355. [CrossRef] 
32. Nagy, F.; Tóth, Z.; Nyikos, F.; Forgács, L.; Jakab, Á.; Borman, A.M.; Majoros, L.; Kovács, R. In Vitro and In Vivo Interaction of Caspofungin with Isavuconazole against Candida Auris Planktonic Cells and Biofilms. bioRxiv 2021, 434267. [CrossRef]

33. Villanueva-Lozano, H.; Treviño-Rangel, R.D.J.; González, G.M.; Ramírez-Elizondo, M.T.; Lara-Medrano, R.; Aleman-Bocanegra, M.C.; Guajardo-Lara, C.E.; Gaona-Chávez, N.; Castilleja-Leal, F.; Torre-Amione, G.; et al. Outbreak of Candida auris infection in a COVID-19 hospital in Mexico. Clin. Microbiol. Infect. 2021. [CrossRef]

34. Flanagan, S.; Goodman, D.B.; Jandourek, A.; O’Reilly, T.; Sandison, T. Lack of Effect of Rezafungin on QT/QTc Interval in Healthy Subjects. Clin. Pharmacol. Drug Dev. 2019, 9, 456-465. [CrossRef] [PubMed] 